\title{
DESIGNING AUDITORY COLOR SPACE FOR COLOR SONIFICATION SYSTEMS
}

Dominik Osinski

\author{
Norwegian University of Science and Technology \\ Department of Electronic Systems, \\ Trondheim, 7491, Norway \\ dominik.osinski@ntnu.no
}

\author{
Helene Midtfjord \\ Norwegian University of Science and Technology \\ Department of Computer Science \\ Gjøvik, 2815, Norway \\ helene.midtfjord@ntnu.no
}

\author{
Dag Roar Hjelme \\ Norwegian University of Science and Technology \\ Department of Electronic Systems \\ Trondheim, 7491, Norway \\ dag.hjelme@ntnu.no
}

\section{ABSTRACT}

Designing of color sonification systems provides a possibility of contribution to various fields ranging from rehabilitation of visually impaired through color perception, multisensory art experience to consciousness studies. The design process itself requires understanding and integrating knowledge from many difficult and inherently different branches of science and the resulting sonification method will be highly dependent on the purpose of the system. We present work in progress on designing and experimental verification of color sonification method that will be implemented in Colorophone - a wearable assistive device for the visually impaired, which enables perception of the information about color through sound. Although our system shows promising results in color and object recognition, we would like to enhance the existing color sonification method by designing a framework for experimental verification of our color sonification algorithm. The goal of this paper is therefore to briefly describe our way of thinking in order to provide the basis for the discussion.

\section{INTRODUCTION}

Our interest in designing intuitive color sonification algorithms is directly related to development of Colorophone - a visual-to-auditory sensory substitution device (SSD) [1]. The main goal of the Colorophone project is to develop an affordable, wearable SSD which will enhance cognitive capabilities of visually impaired by providing auditory information about color and distance. Color sonification

\section{(c) (i) (을} Attribution - Non Commercial 4.0 International License.

The full terms of the License are available at

http://creativecommons.org/licenses/by-nc/4.0/
Patrycja Bizon

\author{
Jagiellonian University \\ Institute of Psychology \\ Ingardena 6, \\ Kraków, 30-060, Poland \\ patrycja.bizon@gmail.com
}

\author{
Michat Wierzchoń \\ Jagiellonian University \\ Institute of Psychology \\ Ingardena 6, \\ Kraków, 30-060, Poland \\ michal.wierzchon@uj.edu.pl
}

systems proved to enhance object recognition and orientation of visually impaired as documented in [2],[3],[4]. Although speaking color monitors are commercially available, coding colors as sound provides much faster and language independent way of delivering the information to users. It also enables active user engagement in the process of scanning of the environment, and development of new sensorimotor contingencies by integration of movement and sound-coded visual information into one multisensory experience. If we looked closer on necessary elements for building a color sonification system, we would conclude that current developments in consumer electronics such as mobile phones, camera technology, and bone conductive headphones are at the level which enables designing SSDs that provide real-time color to sound conversion. The missing element is an intuitive color sonification method.

\section{COLOR SONIFICATION}

Since the goal of the color sonification is to convert information from visual to auditory channel, which are inherently different, we believe that the necessary preliminary step is to specify the function of such a conversion system. In SSDs used for visual rehabilitation of the visually impaired the function of color sonification algorithms is to provide an intuitive information about color by sound. Such systems should therefore be focused on the usability and at the same time provide continuity between different sensory modalities while avoiding sensory overload and limiting interference with other perceptual functions [5]

\subsection{What can we learn from existing systems?}

The existing color sonification methods used in SSDs can be divided into two categories: the first category contains systems which use direct association between color category and 
presented sound [2],[6],[7], for example red color is coded by the sound of choir. In other words, every color is coded by an associated sound, which imposes strict color categorization and step transition between sounds corresponding to colors. The second category of systems uses basic color components associated with sound components [3],[4],[8]. In such systems, auditory color representation is constructed from many sound components, which are merged into one auditory stimulus. The latter category gives significantly better results in experiments related to color recognition, topping out at $98 \%$ of correct answers on the task of identifying 14 colors. Therefore we decided to use the approach described in the second category while designing new color sonification methods. Overview of color sonification methods together with corresponding experimental results are summarized in [3],[4].

\subsection{Mapping sensory components}

In order to associate one sensory modality (color) with another (sound) we have to take into consideration many factors like what the relationship between color and sound components should be, number of used color components, differences in perceptual characteristic of each sensory channel, cross-modal correspondences and finally, which sounds should we use.

\subsubsection{Number of color components}

While thinking about the number of basic color components we should remember that if this number will be too large it could be difficult for the naïve user of the system to remember and recognize all the sounds associated with color components. If the number of basic color components will be too low the user will not have necessary variety in the auditory signal to be able to recognize color change. Our preliminary experiments indicate that although 4 color components (red, green, blue and white) allowed very good auditory color recognition for 14 tested colors (black, white, red, pale red, green, pale green, blue, pale blue, yellow, pale yellow, violet, pale violet, cyan, pale cyan), the recognition of colors in vicinity of yellow (orange, olive green) remains challenging. Since the yellow component is central in opponent process theory [9] and yellow-blue axis is present in many advanced color spaces we consider the yellow channel to be necessary in our color sonification design. Black remains a special color component, because the information about this color, which effectively means lack of any light is conveyed by silence lack of any sound. Definition of five color components plus black strongly reminds of color component definition from Natural Color System (NCS) [10].

\subsubsection{Psychophysics}

Since senses of sight and hearing show different psychophysical characteristics, we implemented inverted Stevens's power law [11] for auditory channel in order to compensate for non-linear response of the human auditory system. The information about the color intensity is preprocessed by the inverted Stevens's power law function which then is annulated by the influence of the human auditory system.

\subsubsection{Cross-modal correspondences}

Cross-modal correspondences are natural associations between different sensory modalities. Although finding an universal mapping remains ambiguous, we can utilize existing research results as a guideline in designing color sonification method. The first intuitive mapping between a color component and a sound component would be the mapping of the intensity of the color stimuli to the intensity of the sound stimuli. The more intensive color will be associated with the sound of higher volume. We chose to associate color components to corresponding sound frequencies on basis of pitch-croma relationship described in [8].

\subsubsection{Sounds associated with color components}

While choosing sound components corresponding to color components, we used the following guidelines: the sounds should be calibrated in amplitude corresponding to maximal color intensity in order to provide equal loudness for every sound component, perceptually equally spaced in the frequency domain and be associated with colors on basis of chosen cross-modal correspondences i.e. blue - low pitch, green - middle pitch, yellow - high pitch, red - high pitch, white - white noise. Since we know which sound pairs will be presented at the same time we can decide if we will present consonant or dissonant pairs of sounds at the same time. For the first version of the system all sounds besides white noise are pure sine tones.

\subsection{Color spaces}

There are numerous color spaces which define the conventions of coding information about color by numerical values. CIELAB and CIELUV are often used, uniform color spaces based on opponent process theory. However CIELAB does not have focal red, blue or green any close to the corresponding color axis, and CIELUV has the biggest deviation from axes for green, yellow and red color components [9]. Focal colors are the best example of a given color category [3]. While designing auditory color space on basis of previous considerations, we need to use a color space based on opponent process theory, where color axes are as close as possible to focal red, yellow, green and blue. We propose to call the color space equipped with the features described above as RYGBW. Possible candidates for being a prototype for developing RYGBW, which meet our requirements, are YCiCii [9] and oRGB [12] color spaces.

\subsection{Auditory color space}

Since the suggested RYGBW color space will be the base for experimental evaluation of color sonification method it does not have to be calibrated in terms of perceptual color distance. Non-linearities in color perception will be mapped by the experiments and could be minimized by an iterative calibration process. We have to remember that the iterative mapping of sound components to color components will compensate for non-linearities in both visual and auditory channels, which is positive for enhancing color sonification algorithms for SSDs. However this compensation process makes research of color perception limited to relative comparisons between participants or participant groups. 


\section{EXPERIMENTAL VERIFICATION}

The main technical part of the system is a software framework developed for experimental verification of color sonification method. It allows automated presentation of test data, logging and postprocessing of the results. A short video which presents our software framework can be found at https://s.ntnu.no/sonification

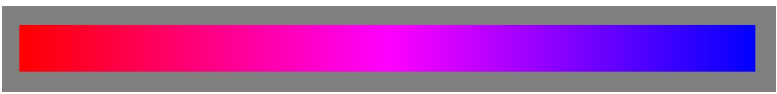

Figure 1: Example of a stripe presenting transition path from red to blue.

\subsection{Experimental procedure}

In order to assure consistency in representation of color stimuli we use an EIZO monitor equipped in build-in color calibration system. Stimuli are presented on a standardized mid-grey background in a dark room. The mid-gray color fills up the whole background on the test monitor. The experiment consists of showing the participant a colorful stripe (Fig. 1). The stripe shows color for one of the basic color transition paths (for example from red to blue through violet). At the same time the system plays a multicomponent sound, where the sound components correspond to red and blue color components. The task of the participant is then to choose the point on the colorful stripe which participant associates with the presented sound. When the participant clicks on the stripe the chosen color is presented in form of rectangle in the middle of the screen in order to eliminate color illusions (Fig. 2.). The participant has then a possibility to correct the choice by clicking on the rectangle or to go to the next trial by clicking below the rectangle. The next trial contains the same colorful stripe but sound components corresponding to red and blue have different amplitude than previously. After performing the whole experiment for one transition path the participant repeats trials with the rest of the transition paths.

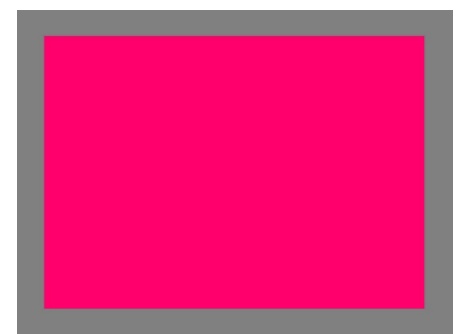

Figure 2: Chosen color presented with grey background to eliminate color illusions.

\subsection{Result analysis}

The proposed method for evaluation of color sonification algorithms enables comparison of numerical RGB values used for sound generation with RGB values chosen by participants. This allows quantitative evaluation of errors in colors picking on basis of auditory signals, which can be used in iterative method for optimization of color sonification algorithms. However, if we assure proper calibration of amplitudes of auditory signals, even the results from the first experimental round can be potentially used to identify differences in color perception between participants.

\section{DISCUSSION AND FUTURE WORK}

We believe that the system for evaluation of color sonification method described in this paper will allow enhancing our existing color sonification method. The enhanced method will then be implemented in the Colorophone SSD and utilized in developing and evaluating of a wearable electronic travel aid for visually impaired as well as in consciousness research in the project "Cognitive and Neural Plasticity and the Subjective Experience. Interdisciplinary Analysis of Sensory Substitution", where the sonification method is used in prolonged training so to induce subjective color perception through audition. Although the beta version of our system is functional, there still are some design challenges that need to be addressed. Which RYGBW color space should we use? Which sound frequencies should be associated with color components? Should the sound pairs be consonant or dissonant? How can we ensure repeatability of stimuli for every participant? The proposed system has been designed to improve our sonification method, however the usage of this system is not limited to design of SSDs. Color research, in spite of growing evidence from neuroscientific studies still remains a place for intensive universalist-relativist debate. Using independent sound variable for evaluation of color perception independently from language constraint seems to be a very interesting path to investigate individual differences in color perception in humans. Development of a web-based system similar to the one described here will allow easy verification of cross-cultural differences in color perception.

\section{ACKNOWLEDGMENT}

This work was supported by the National Science Centre, Poland, grant OPUS (2016/23/B/HS6/00275) given to Michał Wierzchoń.

\section{REFERENCES}

[1] http://www.colorophone.com/

[2] G. Bologna, B. Deville, and T. Pun, "Sonification of Color and Depth in a Mobility Aid for Blind People," in Proceedings of the International Conference on Auditory Displays (ICAD), Washington, D.C, USA, 2010, pp. 9-13.

[3] G. Hamilton-Fletcher and J. Ward, "Representing colour through hearing and touch in sensory substitution devices," Multisens Research, vol. 26, pp. 503- 532, January 2013.

[4] D. Osiński and D. R. Hjelme, "A Sensory Substitution Device Inspired by the Human Visual System," in Proceedings of the 11th International Conference on Human System Interaction (HSI), Gdansk, Poland, 2018, pp. 186-192.

[5] A. Kristjansson, A. Moldoveanu, O. I. Johannesson, O. Balan, S. Spagnol, V. V. Valgeirsdottir, and R. Unnthorsson, "Designing sensory-substitution devices: Principles, pitfalls and potential," Restorative Neurology and Neuroscience, 34(5), pp.769-787, September 2016.

[6] S. Cavaco, J. T. Henriques, M. Mengucci, N. Correia, and F. Medeiros, "Color Sonification for the Visually Impaired," Procedia Technology, vol. 9, pp. 1048-1057, 2013.

[7] S. Abboud, S. Hanassy, S. Levy-Tzedek, S. Maidenbaum, and A. Amedi, "EyeMusic: Introducing a 'visual' colorful experience for the blind using auditory sensory 
substitution," Restor. Neurol. Neurosci., vol. 32, no. 2, pp. 247-257, January 2014.

[8] G. Hamilton-Fletcher, J. Ward, and T. D. Wright, "CrossModal Correspondences Enhance Performance on a Colour-to-Sound Sensory Substitution Device," Multisens. Research, pp. 1-27, February 2016.

[9] N. Moroney, "The opposite of green is purple?" in Proceedings of IS\&T/SPIE Electronic Imaging, San Jose, USA, 2009, Vol. $724172410 \mathrm{~N}-(1-7)$.

[10] https://ncscolour.com/

[11] S. S. Stevens, "On the psychophysical law.," Psychological Review, vol. 64, no. 3, p. 153, June 1957.

[12] M. Bratkova, S. Boulos, and P. Shirley, "orgb: A practical opponent color space for computer graphics." Computer Graphics and Applications, IEEE, 29(1):42-55, Jan.-Feb. 2009. 